\title{
Balkanologie
}

Balkanologie Revue d'études pluridisciplinaires

Vol. XI, $n^{\circ} 1-2$ | 2008

Volume XI Numéro 1-2

\section{Les langues moins répandues en Grèce : état des lieux}

Evangelia Adamou (Sous la direction de), Le nom des langues II: Le patrimoine plurilingue de la Grèce (2008).

\section{Nicolas Trifon}

\section{OpenEdition}

\section{Journals}

Édition électronique

URL : http://journals.openedition.org/balkanologie/1593

DOI : $10.4000 /$ balkanologie. 1593

ISSN : 1965-0582

Éditeur

Association française d'études sur les Balkans (Afebalk)

Référence électronique

Nicolas Trifon, «Les langues moins répandues en Grèce : état des lieux », Balkanologie [En ligne], Vol. $\mathrm{XI}, \mathrm{n}^{\circ} 1-2$ | 2008, mis en ligne le 31 décembre 2008, consulté le 17 décembre 2020. URL : http:// journals.openedition.org/balkanologie/1593; DOI : https://doi.org/10.4000/balkanologie.1593

Ce document a été généré automatiquement le 17 décembre 2020.

(c) Tous droits réservés 


\section{Les langues moins répandues en Grèce : état des lieux}

Evangelia Adamou (Sous la direction de), Le nom des langues II: Le patrimoine plurilingue de la Grèce (2008).

\section{Nicolas Trifon}

1 Cinq auteurs grecs se sont penchés sur le nom et la situation actuelle des langues moins répandues dans leur pays: Stamatis Beis sur l'aroumain, Eleni Botsi sur l'arvanitika, Evangelia Adamou sur l'arménien, Georges Drettas sur le gréco-pontique, Irène Sechidou sur le romani, E. Adamou et G. Drettas sur le slave. Il s'agit du deuxième tome de la série «Le nom des langues » intitulé Le patrimoine plurilingue de la Grèce, un livre à la fois pertinent (les auteurs sont des spécialistes des domaines abordés), riche en informations, indispensables pour saisir la complexité des situations, et édifiant pour ce qui est de la politique linguistique menée par l'Etat grec.

2 Le turc, c'est-à-dire la langue de la seule catégorie des citoyens grecs disposant du statut de minorité, à titre de musulmans, est absent pour des raisons techniques: l'article n'est pas arrivé à temps (p. 16).

3 Les langues abordées sont transmises oralement, dans un cadre familial, n'ont pas de statut officiel, n'ont pas été standardisées, ne sont pas enseignées et ne permettent pas de mobilité sociale aux locuteurs, précise-t-on dans l'introduction (p. 16-17). Il y a cependant une petite exception, l'arménien, enseigné à Athènes (350 élèves) par des professeurs relevant de l'Education nationale grecque. Le nombre de ses locuteurs est restreint, environ 20.000. Certains sont les descendants des réfugiés des années 1919-1923 à Thessalonique, d'autres, la plupart, sont arrivés depuis 1990 de la République ex-soviétique d'Arménie (p. 72). «En Grèce, l'arménien n'a pas le statut de langue minoritaire alors que sa présence est parmi les mieux acceptées par les pouvoirs publics ou par la communauté grecque en général. » (p. 71.) De ce point de vue aussi l'arménien constitue une exception, vraisemblablement parce que cette langue ne représente pas un enjeu sur le plan national.

4 Les locuteurs du gréco-pontique (provenant des territoires situés autour de le mer Noire (Pont Euxin) sont nettement plus nombreux. Leur établissement sur le territoire 
grec remonte à l'échange de populations avec la Turquie en 1923. Une nouvelle vague est arrivée dans les années 1990 en provenance des anciennes républiques soviétiques. Comme tous les dialectes de l'ensemble grec oriental, le gréco-pontique présente suffisamment de différences pour que l'on puisse parler de langue pontique, écrit G. Drettas (p. 75). Chez ses locuteurs, la question des langues n'est pas conflictuelle, poursuit-il en rappelant que, face aux difficultés auxquelles ces déplacés se sont retrouvés confrontés, la langue qui avait de l'importance était celle du pays où ils venaient d'arriver (p. 88). En tout cas, «le grec pontique n'a jamais posé de problème lié à l'identité nationale» (p. 85) et les initiatives en sa faveur ont été peu suivies (p. 86-87). S'agissant officiellement d'un dialecte, son introduction à l'école n'a pas été envisagée.

5 A tous point de vue, la situation de l'arvanitika, de l'aroumain et du slave est différente. Aux yeux de nombre de Grecs, ce sont des «dialectes grecs » en quelque sorte. Le pensent-ils sous l'influence des arguments officiels, qui sont fondés souvent sur des hypothèses invérifiables et qui cherchent à prouver qu'il s'agit de variétés historiques plus ou moins éloignées du grec? Pas forcément, selon E. Adamou, qui estime qu'il s'agit surtout de l'idée bien enracinée selon laquelle « il y a correspondance naturelle d'une langue à une identité nationale». Dans les Balkans, "l'idéologie linguistique dominante rattache toute langue de tradition orale, non standardisée, à une langue standard (...), à une langue nationale, et, partant, intègre la population concernée à une identité nationale ou ethnique ", ce qui "constitue une source de tension entre les Etats voisins », rappelle E. Adamou, en insistant sur le fait que, « en Grèce, à l'exception du romani, toutes les autres langues s'apparentent à des langues nationales dans les autres Etats de la région » (p. 21-22). Pour ma part, je ne la suivrai pas tout à fait dans cette explication. Des situations similaires se présentent aussi dans les autres pays du Sud-Est européen sans que les locuteurs des langues nationales perçoivent celles parlées par leurs compatriotes «minoritaires" comme des « dialectes ». Le turc, le grec ou le hongrois ne sont pas perçus comme des dialectes du bulgare en Bulgarie, de l'albanais, en Albanie ou du roumain en Roumanie.

6 Les rapports entre les langues moins répandues de Grèce et celles en usage dans les Etats voisins ne sont pas expressément évoqués par les auteurs. Leurs contributions ne permettent pas moins d'en saisir la complexité et de comprendre le comportement souvent contradictoire des locuteurs des langues minoritaires en Grèce. Bien placés pour savoir que, en dernière instance, se sont les politiques et non les linguistes qui décident en la matière, les auteurs du livre se gardent bien de se prononcer en termes catégoriques sur le statut du slave, de l'aroumain ou de l'arvanitika. Ils fournissent en revanche suffisamment d'indications pour que le lecteur puisse envisager ces langues comme des réalités vivantes, donc en évolution constante, au-delà des stéréotypes dépréciatifs ou laudatifs circulant sur leur compte.

7 La zone dans laquelle on parle le slave, la partie nord-est de la Macédoine en Grèce et les Rhodopes grecques, est contiguë à la Bulgarie et à la République de Macédoine dont les frontières actuelles ont été établies pour l'essentiel en 1913. Selon des « estimations optimistes ", la slavophonie méridionale compterait quelque 200.000 locuteurs, de confession chrétienne (grecque orthodoxe) et musulmane (p. 126). Les premières implantations slaves dans ces régions remonte aux VIe-VIIe siècles (p. 112).

8 Issu du dialecte tosque (sud de l'Albanie), l'arvanitika est parlé par quelque 95.000 personnes (p. 60). Sa présence en Grèce, dans des enclaves éloignées pour la plupart de 
l'aire albanophone, remonte aux mouvements de population entre la fin de l'ère byzantine et les XVIIe-XVIIIe siècles (p. 48-49) . De confession chrétienne (grecque orthodoxe), les locuteurs de l'arvanitika se sont retrouvés au cœur du premier Etat grec indépendant en 1821.

Attestés pour la première fois au Xe siècle dans la région, les Aroumains sont présents traditionnellement en Grèce du Nord mais aussi dans le Sud albanais et en République de Macédoine. Leur langue, qui remonte à l'administration romaine des Balkans, n'est pas issue du roumain " comme un lecteur contemporain pourrait l'imaginer ", mais à l'instar d'autres parlers romans de la région a connu « un développement indépendant sous forme orale » et "n'a jamais été standardisé » (p. 31). La Bulgarie et la Serbie séparent les aires aroumanophone et roumanophone qui ne sont entrées en contact qu'au dernier tiers du XIXe siècle.

10 Tout en mettant en évidence la diversité des parlers regroupés sous les noms de slave, arvanitika et aroumain, les auteurs font état de nombreux traits communs qui rendent l'intercompréhension entre les locuteurs de chacune de ces langues facile (p. 126, 60 et 36). A ce propos, les signataires de la contribution sur le slave relèvent les effets pervers tant de la sous-estimation que de la surestimation des différences au demeurant bien réelles entre les variétés d'une langue ou à l'intérieur d'un groupe de langues. Le premier cas de figure est illustré par le traitement comme un tout du " groupe roman balkanique » et du « roumain » ou encore des variantes diachroniques et synchroniques de la langue grecque (p. 123-124). La séparation opérée entre le bulgare et le macédonien après la Seconde Guerre illustre par excellence à leurs yeux le deuxième cas. Il existe un « degré important d'intercompréhension entre la plupart des dialectes bulgares et macédoniens ", concluent-ils, tout en faisant par ailleurs état des " petits blocages qui, une fois accumulés en discours, empêchent l'intercompréhension totale [entre les locuteurs des diverses variétés slaves de Grèce] alors que sur le plan structurel les similarités sont nettement présentes » (p. 125).

11 La prise en compte du degré d'intercompréhension peut en effet se révéler précieuse dans toute une série de situations bloquées aussi bien par les interprétations divergentes émises par les linguistes eux-mêmes sur les similitudes et les différences structurelles entre des langues ou des variétés de langues apparentées que par les spéculations d'ordre historique ou plutôt génétique qui circulent à ces sujets. Je pense surtout aux rapports entre le roumain et l'aroumain. Dans les faits, les aroumanophones et leurs interlocuteurs roumanophones peuvent le vérifier à tout instant, il y a un très faible degré d'intercompréhension entre les deux. Le phénomène ne s'explique pas seulement par la forte influence du grec moderne sur le lexique aroumain, rappelée par S. Beis (p. 36), le suivi par un grécophone d'une discussion en aroumain étant encore plus improbable. A eux seuls, ces faits, qui sont mis en évidence par l'application d'un critère somme toute empirique, ne permettent évidemment pas de décréter que l'aroumain est une langue à part. Ils ne sont pas moins incontournables, et en faire abstraction, se serait faire fi des réalités.

12 Plus précisément, se serait faire fi d'un aspect des réalités parmi bien d'autres. Le fait que, par exemple, l'aroumain ne soit pas simplement un dialecte historique extraterritorial du roumain, donc qu'il pourrait être traité de manière indépendante, ou que les parlers slaves de Grèce soient si proches de ceux de la Bulgarie et la République de Macédoine voisines, ne saurait peser lourd par rapport à tout une autre série de faits non moins incontournables: dans le contexte grec, les locuteurs de ces 
parlers, une petite minorité mise à part, n'aspirent pas à les ériger en langues tout court ou à les aligner sur d'autres langues nationales. La complexité des raisons qui les conduisent à adopter cette attitude ressort très bien des contributions portant sur le slave et à l'arvanitika .

En effet, l'histoire régionale qui a engendré la configuration nationale grecque moderne, la résistance des institutions et des mentalités aux changements y compris dans le contexte de l'Union européenne (p. 65), l'effet dissuasif de la répression pratiquée par le passé, sous la dictature de Metaxa notamment, n'expliquent pas tout. Il y a aussi, par exemple, le penchant des locuteurs des petites langues, isolées de surcroît, conduits par les circonstances à utiliser celle de la majorité, de considérer leur parler comme un bien à eux, relevant en quelque sorte de l'intime que l'on hésite et parfois on se refuse à exhiber. Les slavophones, chrétiens comme musulmans, n'appellent-ils pas leur langue (nash-ta) « la notre »? (P. 118.) E. Adamou et G. Drettas avancent l'explication suivante : «l'expression notre langue ne constitue pas seulement un processus de masquage d'une réalité problématique, mais elle souligne l'enracinement de la langue dans un espace villageois dont elle ne représente que l'un des attributs » (p. 119).

«Pour la plupart des locuteurs arvanites, il s'agit d'un code local acquis seulement par les initiés avec une fonction exclusivement interne plutôt que d'une langue "comme les autres ", écrit E. Botsi, qui rapporte l'étonnement provoqué par le contact avec les immigrés arrivés d'Albanie dans les années 1990. Dans les discussions quotidiennes, on évite de citer le terme "arvanitika", en recourrant aux stratégies d'omission", poursuit-elle, tout en rappelant par ailleurs l'expression stéréotypée grecque « Ne me parle pas en arvanitika $=$ Ne me parle pas dans une langue incompréhensible !» (p. 55-56).

$15 \mathrm{La}$ « subordination » et la " dépréciation de soi » constatées chez les « locuteurs finaux (terminal speakers) de l'arvanitika» (p. 68) s'expliquent selon E. Botsi par « le refus de l'Etat grec d'admettre un espace public pour les citoyens hétéroglottes et hétérodoxes » (p. 59). Cela vaut également pour les locuteurs du slave et de l'aroumain, langues dont l'avenir semble tout aussi compromis. La situation des Turcs, comme celle de bon nombre de Slaves (Pomaques) et de Roms de confession musulmane, est différente. Aux termes du traité signé par la Grèce et la Turquie à Lausanne en 1923, les musulmans de Thrace occidentale, la région exempte de l'échange obligatoire de populations, ont une existence légale en Grèce et un enseignement en turc est dispensé dans les écoles publiques de ce pays, notamment depuis 1954 (p. 109). En revanche, l'existence d'une minorité turque n'est pas reconnue. Parmi les effets non recherchés induits par ces dispositions contradictoires il y en a au moins deux qui méritent d'être relevées. D'une part, en se déclarant musulmans, ceux qui ont comme langue première le slave et le romani, langues qui ne sont pas enseignées en Grèce, sont amenés à suivre les cours de turc (et, à une moindre échelle, d'arabe, dans les écoles coraniques). D'autre part, les défaillances de l'enseignement du turc dans le système scolaire grec et les vexations dont font l'objet les «Grecs musulmans » suspectés de nationalisme turc rendent très attractive la Turquie voisine auprès de ces derniers qui s'y rendent par exemple volontiers pour poursuivre leurs études dans de meilleures conditions (131). En Thrace occidentale, des pans entiers de la population échappent ainsi de fait sur certains points à l'autorité de l'Etat grec. Efficace face aux Arvanites, aux Aroumains et aux Slaves grecs-orthodoxes, la stratégie nationale de l'Etat grec fondée sur le choix du 
critère confessionnel hérité de l'Ancien Régime ottoman et le rejet catégorique du critère ethnolinguistique peu ou prou national dans la définition des minorités semble avoir échoué face à ce qui est en train de prendre la forme d'un pôle turc et/ou musulman. 